Article type: Original Research Paper

\title{
Highly Efficient Selective Metamaterial Absorber for High-Temperature Solar Thermal Energy Harvesting
}

\author{
Hao Wang, ${ }^{1}$ Vijay Prasad Sivan, ${ }^{2}$ Arnan Mitchell, ${ }^{2}$ \\ Gary Rosengarten, ${ }^{3}$ Patrick Phelan, ${ }^{1}$ and Liping Wang ${ }^{1, *}$ \\ ${ }^{1}$ School for Engineering of Matter, Transport \& Energy, \\ Arizona State University, Tempe, AZ, 85287, USA \\ ${ }^{2}$ School of Electrical and Computer Engineering, Royal Melbourne \\ Institute of Technology, Melbourne, VIC, 3001, Australia \\ ${ }^{3}$ School of Aerospace, Mechanical and Manufacturing Engineering, Royal \\ Melbourne Institute of Technology, Carlton, VIC, 3053 Australia \\ *Corresponding author: Liping.Wang@asu.edu. Tel: 1-404-863-9372
}

\begin{abstract}
In this work, a selective solar absorber made of nanostructured titanium gratings deposited on an ultrathin $\mathrm{MgF}_{2}$ spacer and a tungsten ground film is proposed and experimentally demonstrated. Normal absorptance of the fabricated solar absorber is characterized to be higher than 0.9 in the UV, visible and, near infrared (IR) regime, while the mid-IR emittance is around 0.2. The high broadband absorption in the solar spectrum is realized by the excitation of surface plasmon and magnetic polariton resonances, while the low mid-IR emittance is due to the highly reflective nature of the metallic components. Further directional and polarized reflectance measurements show wide-angle and polarization-insensitive high absorption within solar spectrum. Temperature-dependent spectroscopic characterization indicates that the optical properties barely change at elevated temperatures up to $350^{\circ} \mathrm{C}$. The solar-to-heat conversion efficiency with the fabricated metamaterial solar absorber is predicted to be $78 \%$ at $100^{\circ} \mathrm{C}$ without optical concentration or $80 \%$ at $400^{\circ} \mathrm{C}$ with 25 suns. The performance could be further improved with better fabrication processes and geometric optimization during metamaterial design. The strong spectral selectivity, favorable diffuse-like behavior, and good thermal stability make the metamaterial selective absorber promising for significantly enhancing solar thermal energy harvesting in various systems at mid to high temperatures.
\end{abstract}

Keywords: solar absorbers, metamaterials, optical properties, high temperatures 


\section{Graphic Abstract}
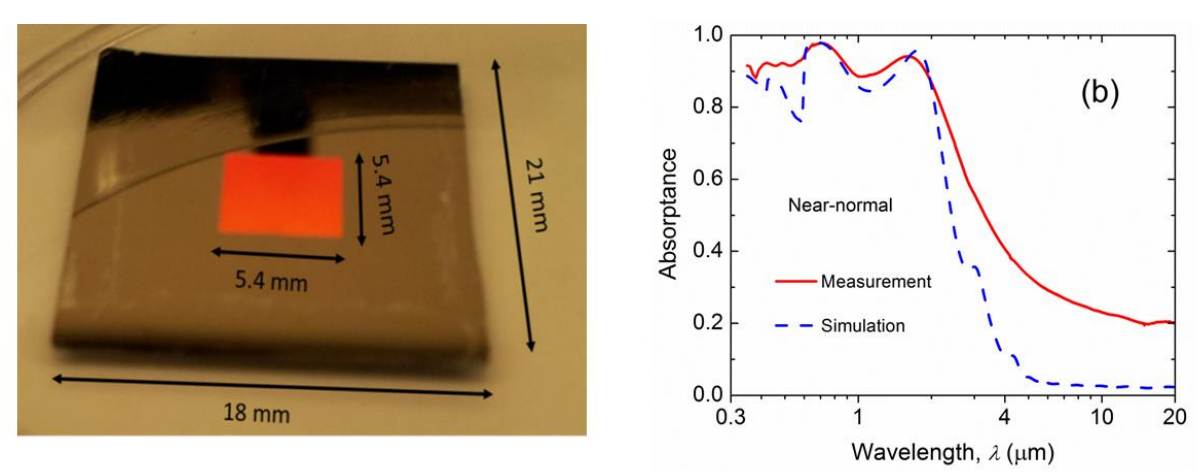

\section{Introduction}

Clean and abundant solar energy has been intensively explored as an alternative to traditional fossil fuels over the past decades. ${ }^{[1]}$ As a key component, absorbers that convert solar radiation into thermal energy greatly affect the performance of various solar thermal systems. An ideal solar absorber should possess an absorptance of unity in the solar spectrum covering UV, visible and near infrared (NIR) to convert most solar radiation into heat, along with zero emittance in the mid-IR regime to minimize energy loss from spontaneous thermal radiation. ${ }^{[2]}$ This spectral selectivity is vital for solar thermal absorbers to achieve high solar-to-heat conversion efficiency. In addition, angular and polarization independence is highly desired for efficient solar absorbers considering the random nature of solar radiation. Excellent thermal stability is also crucial for ensuring solar absorbers to operate properly and efficiently convert solar energy to heat at elevated temperatures over time. Commercially, $\operatorname{TinO}_{\mathrm{x}}{ }^{[3]}$ and Pyromark $^{[4]}$ have been used as solar absorbers for low- to high-temperature applications. Spectrally-selective TinOx coatings could absorb $95 \%$ of incident solar radiation and emit only $4 \%$ thermal radiation, but its performance is optimal only around $100^{\circ} \mathrm{C}$. On the other hand, Pyromark exhibits a nearnormal absorptance above 0.95 at high temperatures around $650^{\circ} \mathrm{C}$, but its thermal emittance is 
also as high as 0.8. Unfortunately, efficient solar absorbers with both spectral selectivity and high-temperature compatibility are still lacking.

Optical metamaterials refer to artificial structures with exotic optical properties that cannot be obtained in naturally occurring materials. ${ }^{[5]}$ Selective absorption has been investigated in metamaterials made of different micro/nanostructures from $\mathrm{GHz}$ to IR spectral regime, including split-ring-resonators, ${ }^{[6,7]}$ fishnet, ${ }^{[8]}$ cut-wires $^{[9,10]}$ and photonic crystals. ${ }^{[11]}$ Recently, film-coupled metamaterials in metal-insulator-metal configurations have been intensively studied as thermal emitters or selective absorbers from visible to NIR spectral regime. Wang and Zhang designed a diffuse-like spectrally-selective thermophotovoltaic (TPV) emitter with 1D tungsten gratings on top of a $\mathrm{SiO}_{2}$ spacer and tungsten substrate, ${ }^{[12]}$ while Zhao et al. modeled the polarization-independent 2D counterparts. ${ }^{[13]}$ Note that selective TPV emitters can also be used as selective solar absorbers due to their spectral selectivity being well-matched to the solar spectrum. Photonic crystals ${ }^{[14,15]}$ and nanoparticles ${ }^{[16-18]}$ were also proposed for selective absorption in the visible and NIR range. Highly efficient solar absorbers require a broad absorption band from visible to NIR range, which could be possibly attained with lossy materials and geometric approaches. Wang and Wang proposed selective metamaterial solar absorbers with multi-sized tungsten patch arrays. ${ }^{[19]}$ Aydin et al. discussed an ultra-thin broadband selective absorber composed of trapezoid gratings. ${ }^{[20]}$ In addition, Lee et al. numerically explored a film-coupled nickel concave grating as a selective absorber for solar thermal energy harvesting. ${ }^{[21]}$

Most of the previous experimental work on metamaterial absorbers has focused on optical characteristics at room temperature. Chen et al. demonstrated an IR selective absorber with film-coupled cross-bar structure at room temperature. ${ }^{[22]}$ Hao et al. investigated a plasmonic 
NIR selective absorber but neglected the effects of temperature on material properties. ${ }^{\text {[23] }}$ However, the temperature-dependent optical properties of novel metamaterial solar absorbers have to be characterized in order to clearly understand the temperature effect as well as thermal stability, which is crucial for ensuring efficient solar energy harvesting at elevated temperatures. ${ }^{[24]}$ Yeng et al. measured the spectral radiance at high temperature for a selective emitter made of tungsten photonic crystal, ${ }^{[25]}$ but the temperature dependence of radiative properties like absorptance or emittance was not investigated experimentally. Liu et al. explored the emittance of a metamaterial thermal emitter at varied temperatures but only in the infrared range. ${ }^{[26]}$ Recently, MIT researchers demonstrated a thermally stable selective solar absorber made of $2 \mathrm{D}$ metallic dielectric photonic crystal structures after $24 \mathrm{hrs}$ heating at $1000^{\circ} \mathrm{C}$, but the temperature-dependent optical properties at high temperatures were not measured. ${ }^{[27]}$ So far, measurements of the optical and radiative properties of selective solar absorbers at elevated temperatures have received little attention. The state of the art of the micro/nanostructured selective solar absorber was recently reviewed by Khodasevych et al. ${ }^{[28]}$

In this work, we report on the spectroscopic characterization at both room and elevated temperatures of a selective metamaterial solar absorber made of a $2 \mathrm{D}$ titanium grating deposited on an $\mathrm{MgF}_{2}$ spacer and an opaque tungsten film, as illustrated in Figure 1a. Tungsten is chosen as the substrate material due to its excellent high-temperature stability and high absorption in the visible and NIR regime. Titanium rather than tungsten in Ref. 19 is selected for the grating materials because of its easiness to pattern in deposition and lift-off processes. $\mathrm{MgF}_{2}$ is used for the dielectric spacer considering its better CTE (i.e., coefficient of expansion) match with tungsten and titanium than other dielectrics like $\mathrm{SiO}_{2}$ to minimize the possibility of thermal cracking at high temperatures. The near-normal specular and hemispherical reflectance is 
measured over a broad spectral range from UV to the mid-IR regime, demonstrating its spectral selectivity. The electromagnetic field distribution obtained from finite-difference time-domain (FDTD) simulation is plotted at absorption peaks to explain underlying mechanism. The effects of oblique incidence, polarization state, as well as temperature up to $350^{\circ} \mathrm{C}$ are further experimentally investigated. Finally, the solar-to-heat conversion efficiency for the metamaterial solar absorber is predicted to show its excellent performance especially at high absorber temperatures.

\section{Sample Fabrication}

The selective metamaterial solar absorber is fabricated with the following procedure. First, $\mathrm{MgF}_{2}$ and tungsten thin films were deposited using e-beam evaporation (Kurt J. Lesker PVD 75) on a silicon substrate. Then, the 2D Ti gratings with period of $600 \mathrm{~nm}$ were fabricated onto the $\mathrm{MgF}_{2} / \mathrm{W}$ coated Si substrate using electron beam lithography by a multi-step exposure scheme on a FEI Nova Nano SEM with NPGS (J. C. Nabity Lithography Systems, Nanometer Pattern Generation System), followed by e-beam evaporation and lift-off process. Figure $1 \mathrm{~b}$ shows the photo of the fabricated metamaterial solar absorber sample with a $5.4 \mathrm{~mm}$ by $5.4 \mathrm{~mm}$ pattern area on a $21 \mathrm{~mm}$ by $18 \mathrm{~mm} \mathrm{Si} \mathrm{wafer.} \mathrm{The} \mathrm{fabricated} \mathrm{grating} \mathrm{patterns} \mathrm{at} \mathrm{the} \mathrm{top} \mathrm{layer} \mathrm{of}$ the metamaterial solar absorber have excellent symmetry in the $\mathrm{x}$ and $\mathrm{y}$ directions as seen from the top-view SEM image in Figure 1c, while a trapezoid shape is observed from the side-view SEM image in Figure 1d, which is typical for metallic gratings patterned from a lift-off process with negative photoresist. The measured geometric parameters are: grating period $\Lambda=600 \mathrm{~nm}$, grating top width $w_{1}=200 \mathrm{~nm}$, bottom width $w_{2}=360 \mathrm{~nm}$, grating height $h=170 \mathrm{~nm}$, and $\mathrm{MgF}_{2}$ 
spacer thickness $t=50 \mathrm{~nm}$. The tungsten layer has a thickness of $200 \mathrm{~nm}$, which is opaque within the spectral region of interests.

\section{Results and Discussion}

3.1. Spectrometric Characterization of Spectral-Normal Reflectance in UV to Mid-IR Regime

The specular reflectance $R_{\lambda}^{\prime}$ of the fabricated solar absorber was measured by a Fourier Transform Infrared (FTIR) spectrometer (Thermo Fisher, iS50) along with a variable-angle reflectance accessory (Harrick Scientific, Seagull) at an incidence angle of $8^{\circ}$ from 0.4 to $20 \mu \mathrm{m}$ in wavelength with a spectral resolution of $4 \mathrm{~cm}^{-1}$ in wavenumber. Due to the excellent geometric symmetry in the $\mathrm{x}$ and $\mathrm{y}$ directions of the sample, the spectrometric measurement was performed with unpolarized waves as the radiative property has negligible polarizationdependence at near-normal direction. The reflectance from 0.4 to $1.1 \mu \mathrm{m}$ in wavelength was measured by an internal Si detector, while an internal DTGS detector was employed at longer wavelengths beyond $1.1 \mu \mathrm{m}$. An aluminum mirror was used as the reference and the measured reflectance is normalized based on the theoretical reflectance of aluminum. The measured reflectance was averaged over three measurements (each with 32 scans) by interchanging the sample and reference to reduce the occasional errors during the measurement. In order to check the uncertainty of FTIR measurements, the reflectance of a reference Si sample (Virginia Semiconductor, Boron doped with resistivity of $60 \mathrm{ohms}-\mathrm{cm}$ ) was measured and compared with its theoretical value, showing the measurement uncertainty within $2 \%$.

The directional-hemispherical reflectance $R_{\lambda}^{\prime}$ and diffuse reflectance were measured in a custom-built 8-inch integrating sphere (Labsphere, IS) at an incidence angle of $8^{\circ}$. An unpolarized monochromatic light from UV to NIR (i.e., $0.35 \mu \mathrm{m}$ to $1.6 \mu \mathrm{m}$ in wavelength) was 
provided by the tunable light source (Newport, TLS-250Q) with a spectral resolution of $10 \mathrm{~nm}$. The light signal was modulated with an optical chopper and measured with a commercial $\mathrm{Si}$ detector (Thorlabs, SM05PD1A) and an InGaAs detector (Thorlabs, SM05PD5A) after lock-in amplification (Oriol, Merlin). A silver mirror was employed as the reference and the measured reflectance is corrected with the theoretical reflectance of silver. The reflectance was averaged from five individual measurements. The hemispherical reflectance was measured without a light trap, while the diffuse reflectance was characterized with a light trap mounted at the specular direction. The measurement uncertainty from the IS measurement was checked to be within $2 \%$ with the same reference Si sample.

Figure 2a shows the room-temperature specular, diffuse, and hemispherical reflectance measured at different wavelengths with an incidence angle $\theta=8^{\circ}$ from both the FTIR and IS measurements. It can be observed that the specular reflectance $R_{\lambda}^{\prime}$ of the metamaterial solar absorber is lower than 0.05 at $0.35 \mu \mathrm{m}<\lambda<0.8 \mu \mathrm{m}$, while $R_{\lambda}^{\prime}$ increases to a maximum value of 0.11 in the NIR range. The low reflectance indicates high absorptance in the solar spectrum, which is desired for highly efficient solar absorbers.

The results of Figure 2a also show that the diffuse reflectance is negligible (less than $0.01)$ at wavelengths $\lambda>650 \mathrm{~nm}$, indicating that the sample is highly specular. This can be explained by the sub-wavelength nature of the metamaterial solar absorber array due to its periodicity. In periodic structures, larger in-plane wavevectors can be obtained with high-order diffracted waves by:

$$
k_{\|, m n}=\left(k_{x, \mathrm{inc}}+\frac{2 \pi m}{\Lambda_{x}}\right) \hat{x}+\left(k_{y, \text { inc }}+\frac{2 \pi n}{\Lambda_{y}}\right) \hat{y}
$$


where $m$ and $n$ are the diffraction orders respectively in the $\mathrm{x}$ and $\mathrm{y}$ directions, while $\Lambda_{x}=\Lambda_{y}=600 \mathrm{~nm}$ is the grating period in both directions for the fabricated metamaterial solar absorber. At normal incidence (i.e., $k_{x, \text { inc }}=k_{y, \text { inc }}=0$ ), when the wavelength of incident waves is larger than the grating period, $k_{\|, m n}$ is larger than the free-space wavelength $k_{\text {inc }}=2 \pi / \lambda$ for all the diffracted waves except for the zeroth order (i.e., $m=n=0$ ), which is the specular reflection component. In other words, all the non-zero diffraction orders are evanescent waves in the subwavelength region, which do not contribute to the far-field diffuse reflection. As a result, the hemispherical reflectance $R_{\lambda}^{\prime}$ from the IS measurement is almost the same as the specular reflectance $R_{\lambda}^{\prime}$ from the FTIR measurement at longer wavelengths beyond $650 \mathrm{~nm}$ with a small difference less than 0.025 mainly due to the different signal-to-noise ratios in both measurements. This highlights the specular behavior of the metamaterial solar absorber when the incident wavelength is larger than the grating period at normal incidence.

On the other hand, when the incident wavelength is smaller than the grating period, the diffuse reflectance becomes significant and increases up to 0.065 around $\lambda=0.5 \mu \mathrm{m}$ due to the non-negligible contribution from higher-order diffracted waves. As a result, the difference between the hemispherical and specular reflectance becomes larger at short wavelengths due to the increased diffuse reflection. Note that from the IS measurement, the diffuse reflectance starts to become negligible at $650 \mathrm{~nm}$, which is not the same as the grating period $\Lambda=600 \mathrm{~nm}$. This is because the reflectance is measured at near normal with $\theta=8^{\circ}$ (i.e.,) instead of perfectly normal incidence. The small nonzero oblique incidence angle results in $k_{x, \text { inc }} \neq 0$, thereby slightly shifting the cutoff wavelength to $\lambda=650 \mathrm{~nm}$. 


\subsection{Spectral-Normal Absorptance/Emittance at Room Temperature}

The spectral-directional absorptance of the metamaterial solar absorber is obtained by $\alpha_{\lambda}^{\prime}=1-R_{\lambda}^{\prime \cap}$ based on the energy balance as the sample is opaque, while the spectral-directional emittance is simply equal to the spectral-directional absorptance according to Kirchhoff's law: $\alpha_{\lambda}^{\prime}=\varepsilon_{\lambda}^{\prime}$. As discussed previously, the metamaterial solar absorber is highly specular at wavelengths $\lambda>650 \mathrm{~nm}$. Thus, the spectral-hemispherical reflectance $R_{\lambda}^{\prime \cap}$ at $\lambda>650 \mathrm{~nm}$ is obtained as the specular reflectance (i.e., $R_{\lambda}^{\prime \cap}=R_{\lambda}^{\prime}$ ) from the FTIR measurement at near-normal incidence, while $R_{\lambda}^{\prime \cap}$ at shorter wavelengths is acquired from the IS measurement. Figure $2 \mathrm{~b}$ shows the characterized spectral absorptance/emittance of the fabricated metamaterial solar absorber under near-normal incidence at room temperature. The metamaterial solar absorber shows absorptance higher than 0.9 within $0.35 \mu \mathrm{m}<\lambda<2 \mu \mathrm{m}$ from the UV to NIR region, and emittance around 0.2 from $6 \mu \mathrm{m}<\lambda<20 \mu \mathrm{m}$ in the mid-IR. Therefore, the spectral selectivity of the metamaterial solar absorber is clearly demonstrated, which is crucial for improving the performance of solar absorbers by maximizing solar absorption and minimizing self-emission loss.

3.3. Underlying Mechanisms for High Ultra-broadband Solar Absorption and Spectral Selectivity FDTD simulation was performed to numerically calculate the spectral-normal absorptance/emittance, which shows excellent agreement with the measurement data in Figure 2b. The FDTD simulation was performed with a commercial package (Lumerical, FDTD Solutions). Optical properties of titanium, $\mathrm{MgF}_{2}$ and tungsten were obtained from Palik's data. ${ }^{[29]}$ The simulation was implemented in a $0.6 \mu \mathrm{m} \times 0.6 \mu \mathrm{m} \times 4 \mu \mathrm{m}$ simulation domain, and the 
wavelength range of interest is from $0.3 \mu \mathrm{m}$ to $20 \mu \mathrm{m}$ with a spectral resolution of $5 \mathrm{~nm}$. Manually refined meshes with a size of $5 \mathrm{~nm}$ in the $\mathrm{x}$ and $\mathrm{y}$ directions and $2 \mathrm{~nm}$ in the $\mathrm{z}$ direction were employed to ensure the numerical convergence. Periodic boundary conditions were applied in the $\mathrm{x}$ and $\mathrm{y}$ directions for normal incidence, while perfect matched layers with reflection coefficient of $10^{-6}$ were placed in the $\mathrm{z}$ direction. A plane wave source was placed at $1.2 \mu \mathrm{m}$ above the structure surface, and the reflectance $R$ was obtained by a frequency-domain power monitor positioned at $0.5 \mu \mathrm{m}$ above the plane wave source. The spectral absorptance was obtained using $\alpha=1-R_{\lambda}^{\prime \cap}$ as the structure is opaque due to the $200 \mathrm{~nm}$ tungsten substrate.

There are two major absorption peaks from the measurement for the metamaterial solar absorber: one at $\lambda=0.68 \mu \mathrm{m}$ with an amplitude of 0.978 due to surface plasmon polariton (SPP), ${ }^{[2,30,31]}$ and the other at $\lambda=1.6 \mu \mathrm{m}$ with an amplitude of 0.941 due to magnetic polariton (MP). ${ }^{[12,13,19,32]}$ SPP is a surface wave due to the collective oscillation of plasmon excited at the interface of two materials with permittivity of $\varepsilon_{1}$ and $\varepsilon_{2}$. Note that the real parts of $\varepsilon_{1}$ and $\varepsilon_{2}$ should have opposite signs, and a SPP is excited when the following dispersion relation is satisfied: ${ }^{[2]}$

$$
k_{\mathrm{SPP}}=\frac{\omega}{c_{0}} \sqrt{\frac{\varepsilon_{1} \varepsilon_{2}}{\varepsilon_{1}+\varepsilon_{2}}}
$$

The dispersion relation theoretically predicts a SPP mode excited for the Ti gratings at $\lambda_{\mathrm{SPP}}=0.68 \mu \mathrm{m}$ with $\theta=8^{\circ}$. It can be observed that the measured absorptance is slightly higher than simulation results at the visible range of $0.4 \mu \mathrm{m}<\lambda<0.6 \mu \mathrm{m}$. This is because the SPP absorption peak wavelength is highly dependent on incidence angles. During the measurement, the light is incident onto the sample at $\theta=8^{\circ}$ with a half-cone angle of $1.5^{\circ}$ or so. Therefore, the 
absorption peak associated with SPP is broadened from the measurement in this visible regime due to the finite solid angle of the incident beam.

On the other hand, MP is the coupling between the incident electromagnetic wave and magnetic resonance inside the structure. To explain the excitation mechanism of MP, the electromagnetic field distribution at the MP resonance is obtained from the FDTD simulation and plotted in Figure 3. The arrows show the electric field vectors and the contour represents the strength of the magnetic field normalized as $\log _{10}\left|H / H_{0}\right|^{2}$, where $H_{0}$ is the incident magnetic field. It can be observed that the electric current forms a loop under the Ti patch, while the magnetic field is greatly enhanced in the local area within the current loop by one order of magnitude higher compared to the incident field magnitude. This is a typical electromagnetic field pattern at MP resonance due to the diamagnetic response inside the grating microstructure, ${ }^{[12,13,19,32]}$ and the strong field confinement explains the high absorption at MP resonance.

The measured emittance in the mid infrared is around 0.20 , which is higher than the 0.04 predicted from the simulation, possibly due to the oxidation of tungsten and titanium during the sample fabrication process. Note that, the metamaterial solar absorber is essentially highly reflective from the metallic components without any resonance absorption in the longwavelength region. By addressing the material oxidation issue, it is expected that, the emittance of the metamaterial solar absorber sample can be further reduced to approach the theoretical value of 0.04 to better minimize the thermal emission energy loss, thereby further improving the solar thermal conversion performance.

\subsection{Angular Behavior of the Selective Metamaterial Solar Absorber}


The optical and radiative properties at oblique angles of a solar absorber are also vital for efficiently harvesting direct sunlight coming from different directions after an optical concentrator. An ideal solar absorber should be diffuse-like with optical and radiative properties independent of direction. Therefore, the specular reflectance of the metamaterial solar absorber was measured by the FTIR spectrometer at several incidence angles $\theta=5^{\circ}, 15^{\circ}, 30^{\circ}$ and $45^{\circ}$ with the variable-angle reflectance accessory. The reflectance was measured separately for transversemagnetic (TM) and transverse-electric (TE) waves with a broadband polarizer (Thorlabs, WP25M-UB) in the visible and NIR regime or the internal wire-grid IR polarizer inside the FTIR.

Figure 4a plots the measured specular reflectance $R_{\lambda}^{\prime}$ for the metamaterial solar absorber at oblique TM incidence. It can be observed that $R_{\lambda}^{\prime}$ at $\lambda<0.7 \mu \mathrm{m}$ is lower than 0.05 for all oblique incidences. Moreover, the reflection dip due to the MP excitation at $\lambda=1.6 \mu \mathrm{m}$ does not shift with increased incidence angle, thanks to the unique direction-independent characteristic of MP resonance. ${ }^{[12,32]}$ When the incidence angle increases for TM incidence, the strength of the incident $\mathrm{H}$ field parallel to the $\mathrm{y}$-direction grating groove does not change. Thus, the strength of the oscillating current loop for MP does not decrease, and the MP resonance strength remains almost unchanged. ${ }^{[13]}$ As a result, the reflectance at the MP wavelength increases little with larger incidence angle.

Figure $4 \mathrm{~b}$ shows the specular reflectance $R_{\lambda}^{\prime}$ of the metamaterial solar absorber at TE oblique incidence. It is found that $R_{\lambda}^{\prime}$ at $\lambda<0.7 \mu \mathrm{m}$ is also lower than 0.05 . The reflectance at MP resonance increases slightly with larger oblique angles. This is because the strength of the incident $\mathrm{H}$ field component parallel to the grating groove in the $\mathrm{y}$ direction decreases as the incidence angle becomes larger for TE waves. As a result, the strength for MP resonance 
decreases and the absorptance drops ${ }^{[13]}$ The reflectance at longer wavelengths in the mid-IR also increases slightly at oblique incidences.

Considering the random nature of sunlight, the reflectance for unpolarized waves, which is averaged from both polarizations, is presented in Figure 4c for different oblique angles. It is observed that when incidence angle $\theta$ changes from $5^{\circ}$ to $30^{\circ}$, the reflectance for unpolarized incidence barely changes with reflectance lower than 0.15 in the visible and NIR regions (i.e., $0.4 \mu \mathrm{m}$ to $2 \mu \mathrm{m}$ in wavelength). The reflectance slightly increases but remains less than 0.2 when $\theta$ further increases to $45^{\circ}$. The measured optical and radiative properties of the fabricated metamaterial solar absorber sample clearly demonstrate the diffuse-like behaviors at both TM and TE polarizations as well as for unpolarized waves.

\subsection{Temperature-Dependent Spectrometric Characterization with FTIR Fiber Optics}

In order to characterize the optical properties of the metamaterial solar absorber at elevated temperatures, an FTIR fiber optics technique as illustrated in Figure 5a was developed for measuring the temperature-dependent specular reflectance $R_{\lambda}^{\prime}$ from the sample mounted inside a home-designed heater assembly with precise temperature control. An FTIR fiber coupler (Harrick, Fibermate2) was employed to couple the FTIR with a VIS-NIR reflection (also called back-scattering) optics fiber bundle (Thorlabs, RP21). A fiber probe with collimating and focusing optics yielded a beam spot with a diameter of $4 \mathrm{~mm}$ onto the sample surface. The normally reflected signal was then collected by the same probe and acquired by the FTIR detectors through optical fibers. The sample was mounted onto a copper disk inside a home-built heater assembly. A thermocouple (Omega, KMTXL-040) was utilized to measure the sample temperature and the temperature was sent to a temperature controller (Omega, CSi8D), which 
modulated the power input to the heater assembly and thereby accurately maintained the sample temperature at the setpoint within $1 \mathrm{~K}$ variation. The FTIR measurements were performed when the desired temperature was stable at least for $20 \mathrm{~min}$. Note that in the NIR spectral regime, the measured reflectance at each wavelength is averaged from 20 neighboring data points to reduce the fluctuation in measured reflectance caused by the low signal-to-noise ratio from the DTGS detector. The measurement uncertainty was found to be smaller than $2.5 \%$ with the reference $\mathrm{Si}$ sample. A 30-nm-thick $\mathrm{SiO}_{2}$ layer was deposited onto the sample surface to protect the metamaterial structures from possible oxidation or chemical reaction in air during sample heating, which would change the desired optical properties and degrade the performance of the metamaterial solar absorber at elevated temperatures.

Figure $5 \mathrm{~b}$ shows the reflectance for the metamaterial solar absorber when the sample temperature increases from room temperature $\left(23.5^{\circ} \mathrm{C}\right)$ to $350^{\circ} \mathrm{C}$ with an interval of $50^{\circ} \mathrm{C}$. It can be seen that the reflectance at wavelengths from $0.4 \mu \mathrm{m}$ to $0.8 \mu \mathrm{m}$ barely changes with increased absorber temperature, indicating good thermal stability of the fabricated metamaterial solar absorber. The reflectance from $0.8 \mu \mathrm{m}<\lambda<2 \mu \mathrm{m}$ decreases with higher temperatures, which might be due to materials chemical changes. Nevertheless, the slight variation of the reflectance is only within 0.025 , and decreased NIR reflectance at higher temperature is actually beneficial for absorbing more solar energy.

\subsection{Efficiency Analysis for Solar Thermal Energy Conversion}

In order to evaluate the performance of the metamaterial as a potential highly efficient solar thermal absorber, the solar-to-heat conversion efficiency is theoretically analyzed. 
Assuming no conduction or convection losses, the conversion efficiency of a solar absorber can be calculated by:

$$
\eta=\frac{\alpha_{\text {Total, } \mathrm{N}} C G-\varepsilon_{\text {Total, }}\left(\sigma T_{\mathrm{A}}^{4}-\sigma T_{\mathrm{sky}}^{4}\right)}{C G}
$$

where $C$ is the concentration factor, $G$ is the heat flux of incident solar irradiation at AM1.5 (global tilt), ${ }^{[33]} T_{\mathrm{A}}$ is the absorber temperature, and $T_{\text {sky }}=20^{\circ} \mathrm{C}$ is the sky temperature. $\alpha_{\text {Total, }}$ and $\varepsilon_{\text {Total, N }}$ are respectively the total normal absorptance and emittance for the solar absorber, which are calculated by:

$$
\begin{gathered}
\alpha_{\text {Total }, \mathrm{N}}=\int_{0}^{\infty} \alpha_{\lambda, \mathrm{N}}^{\prime} I_{\mathrm{AM} 1.5}(\lambda) d \lambda / \int_{0}^{\infty} I_{\mathrm{AM} 1.5}(\lambda) d \lambda \\
\varepsilon_{\text {Total }, \mathrm{N}}=\int_{0}^{\infty} \varepsilon_{\lambda, N}^{\prime} I_{\mathrm{BB}}\left(\lambda, T_{\mathrm{A}}\right) d \lambda / \int_{0}^{\infty} I_{\mathrm{BB}}\left(\lambda, T_{\mathrm{A}}\right) d \lambda
\end{gathered}
$$

where $I_{\mathrm{AM} 1.5}(\lambda)$ is the spectral intensity of solar irradiation at AM1.5 (global tilt), ${ }^{[33]} I_{\mathrm{BB}}\left(\lambda, T_{\mathrm{A}}\right)$ is the spectral blackbody radiative intensity at the solar absorber temperature $T_{\mathrm{A}}$, and $\alpha_{\lambda, N}^{\prime}$ and $\varepsilon_{\lambda, N}^{\prime}$ are respectively the spectral normal absorptance and emittance of the solar absorber measured at room temperature. Note that both $\alpha_{\lambda, N}^{\prime}$ and $\varepsilon_{\lambda, N}^{\prime}$ are taken to be independent of temperature as observed from the temperature-dependent optical characterization. For the calculation of total absorptance, the spectral integration is limited to the wavelength region from $0.35 \mu \mathrm{m}$ to $4 \mu \mathrm{m}$, because our instrument cannot measure optical properties at wavelengths below $0.35 \mu \mathrm{m}$ while the available AM1.5 data only covers wavelengths up to $4 \mu \mathrm{m}$. There is still around $7 \%$ of solar radiation outside of this spectral range mainly in the UV regime. Similarly, the spectral integration for total emittance is performed over wavelengths from 0.35 $\mu \mathrm{m}$ to $20 \mu \mathrm{m}$, again limited by the available measurement data. Note that there is only $4 \%$ 
energy outside this spectral regime mainly in the far infrared for a blackbody with a temperature of $400^{\circ} \mathrm{C}$. Since the metamaterial solar absorber is quite diffuse with oblique angles $\theta<45^{\circ}$ from the directional optical property characterization, the total hemispherical absorptance or emittance can be reasonably approximated by the total normal absorptance or emittance.

Figure 6a plots the conversion efficiency $\eta$ as a function of absorber temperature $T_{\mathrm{A}}$ under 1 sun (i.e., no optical concentration) for an ideal selective surface, the metamaterial solar absorber with optical and radiative properties taken from either measurements or the FDTD simulation, and a black surface. The absorptance for the ideal surface is unity below the cutoff wavelength to maximize absorbed solar radiation, while its emittance is zero beyond the cutoff wavelength to minimize spontaneous thermal emission loss. The cutoff wavelength for the ideal selective solar absorber is optimized at each absorber temperature for maximal conversion efficiency, which represents the upper limit. On the other hand, the black surface has unity absorptance and emittance in the entire spectral regime (i.e., $\varepsilon=\alpha=1$ ), whose conversion efficiency indicates the lowest limit.

It is observed that the conversion efficiency for the metamaterial solar absorber with measured optical properties could reach $78.1 \%$ at the absorber temperature $T_{\mathrm{A}}=100^{\circ} \mathrm{C}$ and monotonically drops to zero at the stagnation temperature of $241^{\circ} \mathrm{C}$, at which no solar thermal energy is harvested. The efficiency for the metamaterial absorber with simulated optical properties shows relatively higher values. Theoretically, the proposed metamaterial absorber could have a conversion efficiency as high as $88.3 \%$ at $T_{\mathrm{A}}=100^{\circ} \mathrm{C}$ and a much higher stagnation temperature of $393^{\circ} \mathrm{C}$. The discrepancy on the efficiency results from larger emittance in the midIR region from the measurement than simulation. The performance of the fabricated 
metamaterial absorber can be further improved to approach the theoretical values after the oxidation issues during the sample fabrication are addressed.

In comparison, a black surface could only convert about $32 \%$ of solar energy to useful heat at $T_{\mathrm{A}}=100^{\circ} \mathrm{C}$, while its efficiency drops quickly to zero at $125^{\circ} \mathrm{C}$, suggesting the great importance of spectral selectivity in enhancing the solar-to-heat conversion efficiency. On the other hand, the efficiency of the metamaterial absorber is about $10 \%$ (with simulated optical properties) or $20 \%$ (with measured data) less than the ideal surface at $T_{\mathrm{A}}=100^{\circ} \mathrm{C}$, mainly due to the larger emittance in the mid-IR around 0.04 (simulated) and 0.20 (measured). The absorptance within the solar spectrum of the metamaterial is also smaller than the ideal by 0.05 to 0.1 , but at low optical concentrations, the self-emission loss determined by the mid-IR emittance plays a major role in determining the amount of harvested solar energy. The difference between the metamaterial absorber and the ideal surface becomes even larger when temperature goes up as the ideal surface maintains high efficiencies of $98.7 \%$ at $200^{\circ} \mathrm{C}$, and $93 \%$ at $400^{\circ} \mathrm{C}$. This is because the cut-off wavelength of the ideal surface is optimized at each temperature, as the blackbody spectrum governed by Planck's law would shift towards shorter wavelengths with higher absorber temperature. On the other hand, the cut-off wavelength for the metamaterial absorber is around $\lambda=2 \mu \mathrm{m}$ or so and does not change with absorber temperature. In fact, the cut-off wavelength of the metamaterial absorber is determined by the MP resonance wavelength, which can be easily tuned with geometric parameters such as grating width. Therefore, for a given absorber temperature required by a particular solar thermal system, the cut-off wavelength as well as the absorptance/emittance spectrum can be optimized during the design and fabrication processes for achieving maximal solar-to-heat conversion efficiency. 
The solar-to-heat conversion efficiency can be further improved with concentrated sunlight. Here, we consider the effect of concentration factor $C$ from 1 to 50 at an absorber temperature $T_{\mathrm{A}}=400^{\circ} \mathrm{C}$ for a medium-temperature application. Note that the thermal energy at $400^{\circ} \mathrm{C}$ carries quite an amount of exergy, and could potentially deliver electricity via heat engines like the Rankine cycle, or solid-state devices such as thermoelectrics and TPV in addition to heating and cooling applications. Figure $6 \mathrm{~b}$ shows that the metamaterial absorber with measured optical properties could harvest $21.5 \%$ of solar energy to useful heat under 5 suns, $57.4 \%$ with 10 suns, and $80 \%$ with 25 suns. For the metamaterial absorber with even lower IR emittance as simulated, the efficiency could be as high as $71 \%$ under 5 suns and $81 \%$ with 10 suns, indicating room for improvement with the current fabricated sample. With greater optical concentrations up to 100 suns, the conversion efficiency of the metamaterial absorber (with optical properties both measured and simulated) saturates towards 90\%. In comparison, the efficiency of the ideal surface slightly increases from $93 \%$ to $98 \%$ at the same temperature from 1 sun to 100 suns, while that of a black surface is improved greatly with higher optical concentration from $0 \%$ at 11 suns and approaching 90\% with 100 suns, suggesting that the spectral selectivity becomes less important at concentration larger than 100 as thermal emission loss becomes negligible with highly concentrated incident solar radiative flux.

\section{Conclusion}

In summary, we have proposed and experimentally characterized the optical and radiative properties of a highly-efficient metamaterial selective absorber for solar thermal energy harvesting at elevated temperatures up to $350^{\circ} \mathrm{C}$. The metamaterial solar absorber is demonstrated to exhibit absorptance higher than 0.9 within the solar spectrum and low emittance of around 0.2 in the mid IR regime. Besides, the measured reflectance shows diffuse-like 
behavior at large oblique incidence, indicating wide-angle and polarization-insensitive high absorption within solar spectrum. Characterization of the temperature-dependent optical properties of the solar absorber sample demonstrated good thermal stability and excellent temperature-insensitivity up to $350^{\circ} \mathrm{C}$. Based on the measured optical properties of the fabricated metamaterial sample, the solar-to-heat conversion efficiency from this solar absorber is predicted to be $78 \%$ at $100^{\circ} \mathrm{C}$ without optical concentration, or $80 \%$ at $400^{\circ} \mathrm{C}$ with 25 suns. By addressing the oxidation issues during fabrication, the efficiency from the metamaterial solar absorber could be increased by a few more percent, indicated by the simulated data. In addition, by carefully optimizing the geometry parameters from the design, the efficiency could be further maximized for a given operating temperature. Insights gained from this work will facilitate the design and optimization of a new class of selective solar absorbers for enhancing solar energy harvesting at medium-to-high temperatures in various systems including but not limited to solar heating and cooling, concentrated solar power, solar thermoelectrics, and solar TPV.

\section{ACKNOWLEDGMENT}

This work was supported by the US-Australia Solar Energy Collaboration - Micro Urban Solar Integrated Concentrators (MUSIC) project sponsored by the Australian Renewable Energy Agency (ARENA). HW and LW would like to thank the partial supports from the National Science Foundation under a CAREER Award (CBET-1454698) and ASU New Faculty Startup fund. The authors acknowledge the facilities, and the scientific and technical assistance of the Australian Microscopy \& Microanalysis Research Facility at RMIT University. 


\section{References}

[1] S. Mekhilef, R. Saidur, A. Safari, A review on solar energy use in industries, Renew. Sust. Energ. Rev., 15 (2011) 1777-1790.

[2] Z.M. Zhang, Nano/microscale heat transfer, McGraw-Hill, New York, 2007.

[3] Solar Brochure of $\mathrm{TinO}_{\mathrm{x}}$, ALMECO Group, http://www.almecogroup.com/en/pagina/16$\underline{\text { solar. }}$

[4] C.K. Ho, A.R. Mahoney, A. Ambrosini, M. Bencomo, A. Hall, T.N. Lambert, Characterization of pyromark 2500 paint for high-temperature solar receivers, J. Sol. Energy Eng., 136 (2014) 014502.

[5] Y. Liu, X. Zhang, Metamaterials: a new frontier of science and technology, Chem. Soc. Rev., 40 (2011) 2494-2507.

[6] R. Marques, J. Martel, F. Mesa, F. Medina, Left-handed-media simulation and transmission of EM waves in subwavelength split-ring-resonator-loaded metallic waveguides, Phys. Rev. Lett., 89 (2002) 183901.

[7] K. Aydin, E. Ozbay, Capacitor-loaded split ring resonators as tunable metamaterial components, J. Appl. Phys., 101 (2007) 024911.

[8] D.Y. Shchegolkov, A. Azad, J. O’Hara, E. Simakov, Perfect subwavelength fishnetlike metamaterial-based film terahertz absorbers, Phys. Rev. B, 82 (2010) 205117.

[9] N. Liu, H. Guo, L. Fu, S. Kaiser, H. Schweizer, H. Giessen, Plasmon hybridization in stacked cut-wire metamaterials, Adv. Mater., 19 (2007) 3628-3632.

[10] H. Tao, N.I. Landy, C.M. Bingham, X. Zhang, R.D. Averitt, W.J. Padilla, A metamaterial absorber for the terahertz regime: Design, fabrication and characterization, Opt. Express, 16 (2008) 7181-7188.

[11] E. Rephaeli, A. Raman, S.H. Fan, Ultrabroadband photonic structures to achieve highperformance daytime radiative cooling, Nano Lett., 13 (2013) 1457-1461.

[12] L.P. Wang, Z.M. Zhang, Wavelength-selective and diffuse emitter enhanced by magnetic polaritons for thermophotovoltaics, Appl. Phys. Lett., 100 (2012) 063902.

[13] B. Zhao, L.P. Wang, Y. Shuai, Z.M. Zhang, Thermophotovoltaic emitters based on a twodimensional grating/thin-film nanostructure, Int. J. Heat Mass Transfer, 67 (2013) 637-645.

[14] I. Celanovic, N. Jovanovic, J. Kassakian, Two-dimensional tungsten photonic crystals as selective thermal emitters, Appl. Phys. Lett., 92 (2008) 193101. 
[15] W. Wang, Y. Cui, Y. He, Y. Hao, Y. Lin, X. Tian, T. Ji, S. He, Efficient multiband absorber based on one-dimensional periodic metal-dielectric photonic crystal with a reflective substrate, Opt. Lett., 39 (2014) 331-334.

[16] M. Yan, J. Dai, M. Qiu, Lithography-free broadband visible light absorber based on a mono-layer of gold nanoparticles, J. Opt., 16 (2014) 025002.

[17] B. Chen, W. Zhang, X. Zhou, X. Huang, X. Zhao, H. Wang, M. Liu, Y. Lu, S. Yang, Surface plasmon enhancement of polymer solar cells by penetrating $\mathrm{Au} / \mathrm{SiO}_{2}$ core/shell nanoparticles into all organic layers, Nano Energy, 2 (2013) 906-915.

[18] H. Wang, K. O’Dea, L.P. Wang, Selective absorption of visible light in film-coupled nanoparticles by exciting magnetic resonance, Opt. Lett., 39 (2014) 1457-1460.

[19] H. Wang, L.P. Wang, Perfect selective metamaterial solar absorbers, Opt. Express, 21 (2013) A1078-A1093.

[20] K. Aydin, V.E. Ferry, R.M. Briggs, H.A. Atwater, Broadband polarization-independent resonant light absorption using ultrathin plasmonic super absorbers, Nat. Commun., 2 (2011) 517.

[21] B.J. Lee, Y.-B. Chen, S. Han, F.-C. Chiu, H.J. Lee, Wavelength-selective solar thermal absorber with two-dimensional nickel gratings, J. Heat Transfer, 136 (2014) 072702.

[22] K. Chen, R. Adato, H. Altug, Dual-band perfect absorber for multispectral plasmonenhanced infrared spectroscopy, Acs Nano, 6 (2012) 7998-8006.

[23] J. Hao, J. Wang, X. Liu, W.J. Padilla, L. Zhou, M. Qiu, High performance optical absorber based on a plasmonic metamaterial, Appl. Phys. Lett., 96 (2010) 251104.

[24] L.P. Wang, Z.M. Zhang, Measurement of coherent thermal emission due to magnetic polaritons in subwavelength microstructures, J. Heat Transfer, 135 (2013) 091505.

[25] Y.X. Yeng, M. Ghebrebrhan, P. Bermel, W.R. Chan, J.D. Joannopoulos, M. Soljačić, I. Celanovic, Enabling high-temperature nanophotonics for energy applications, Proc. Natl. Acad. Sci., 109 (2012) 2280-2285.

[26] X. Liu, T. Tyler, T. Starr, A.F. Starr, N.M. Jokerst, W.J. Padilla, Taming the blackbody with infrared metamaterials as selective thermal emitters, Phys. Rev. Lett., 107 (2011) 045901.

[27] J.B. Chou, Y.X. Yeng, Y.E. Lee, A. Lenert, V. Rinnerbauer, I. Celanovic, M. Soljačić, N.X. Fang, E.N. Wang, S.G. Kim, Enabling ideal selective solar absorption with 2D metallic dielectric photonic crystals, Adv. Mater., (2014). DOI: 10.1002/adma.201403302. 
[28] I. Khodasevych, L. Wang, A. Mitchell, G. Rosengarten, Micro and nanostructured surfaces for selective solar absorption To be submitted, (2014).

[29] E.D. Palik, Handbook of optical constants of solids, Academic press, 1998.

[30] S.A. Maier, Plasmonics: fundamentals and applications, Springer, 2007.

[31] S. Wedge, I.R. Hooper, I. Sage, W.L. Barnes, Light emission through a corrugated metal film: The role of cross-coupled surfaceplasmon polaritons, Phys. Rev. B, 69 (2004) 245418.

[32] L.P. Wang, Z.M. Zhang, Effect of magnetic polaritons on the radiative properties of doublelayer nanoslit arrays, JOSA B, 27 (2010) 2595-2604.

[33] Air Mass 1.5 Spectra, American Society for Testing and Materials (ASTM), Available from: http://rredc.nrel.gov/solar/spectra/am1.5/. 


\section{Figure Captions:}

Figure 1. (a) Structure schematic for proposed metamaterial solar absorber. $\mathbf{K}_{\text {inc }}$ represents the incident wavevector, and the incidence angle $\theta$ is defined as the angle between $\mathbf{K}_{\text {inc }}$ and the surface normal. The plane of incidence (POI) is defined as the $\mathrm{x}-\mathrm{z}$ plane. The $\mathbf{E}$ field is in the POI for transverse magnetic (TM) incidence, while it is perpendicular to the POI for transverse electric (TE) incidence. (b) A photo of the fabricated sample for optical characterization. SEM images of the fabricated absorber sample from (c) top view and (d) side view.

Figure 2. (a) Measured room-temperature specular, diffuse, and hemispherical reflectance of the metamaterial solar absorber. (b) Measured and simulated room-temperature spectral absorptance of the metamaterial solar absorber.

Figure 3. Cross-sectional view of electromagnetic field at the MP resonance wavelength from the FDTD simulation. The arrows represent strength and direction of the electric field, while the contour plot illustrates strength of magnetic field.

Figure 4. (a) Specular reflectance of the metamaterial solar absorber sample measured by FTIR at (a) oblique TM incidences, (b) oblique TE incidences, and (c) unpolarized oblique incidences.

Figure 5. (a) Schematic of the experimental setup for the temperature-dependent FTIR fiber optics measurements for characterizing spectral normal reflectance at elevated temperatures. (b) Specular reflectance of the metamaterial solar absorber measured at elevated temperatures up to $350^{\circ} \mathrm{C}$ with temperature-dependent FTIR fiber optics.

Figure 6. (a) Predicted solar-to-heat conversion efficiencies of an ideal selective surface, the metamaterial solar absorber (with optical properties either measured or simulated), and a black surface as a function of absorber temperature $T_{\mathrm{A}}$ under 1 sun. (b) Solar-to-heat conversion efficiency for all three surfaces as a function of concentration factor $C$ at an absorber temperature of $T_{\mathrm{A}}=400^{\circ} \mathrm{C}$. 

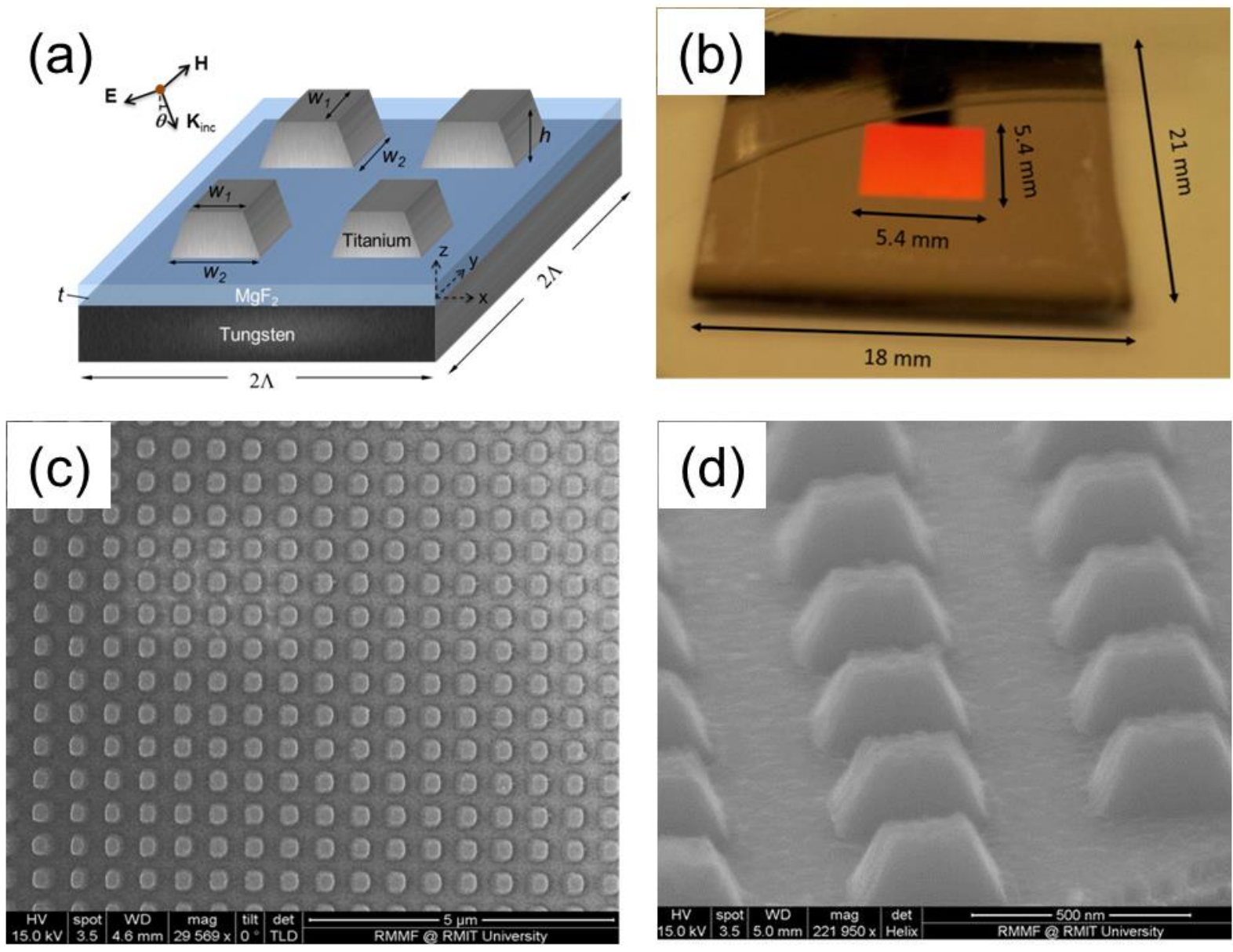

Wang et al., Figure 1 

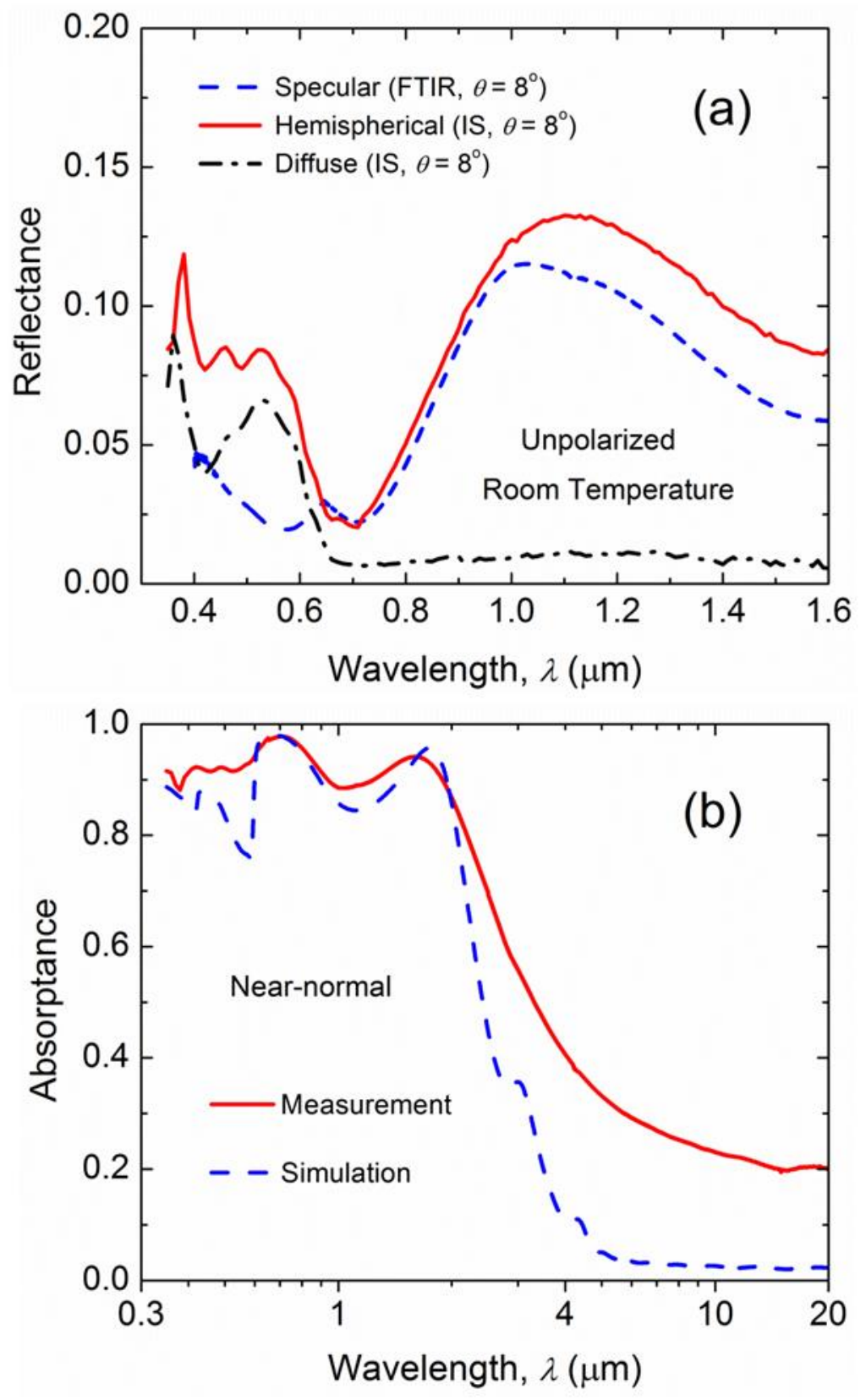

Wang et al., Figure 2 


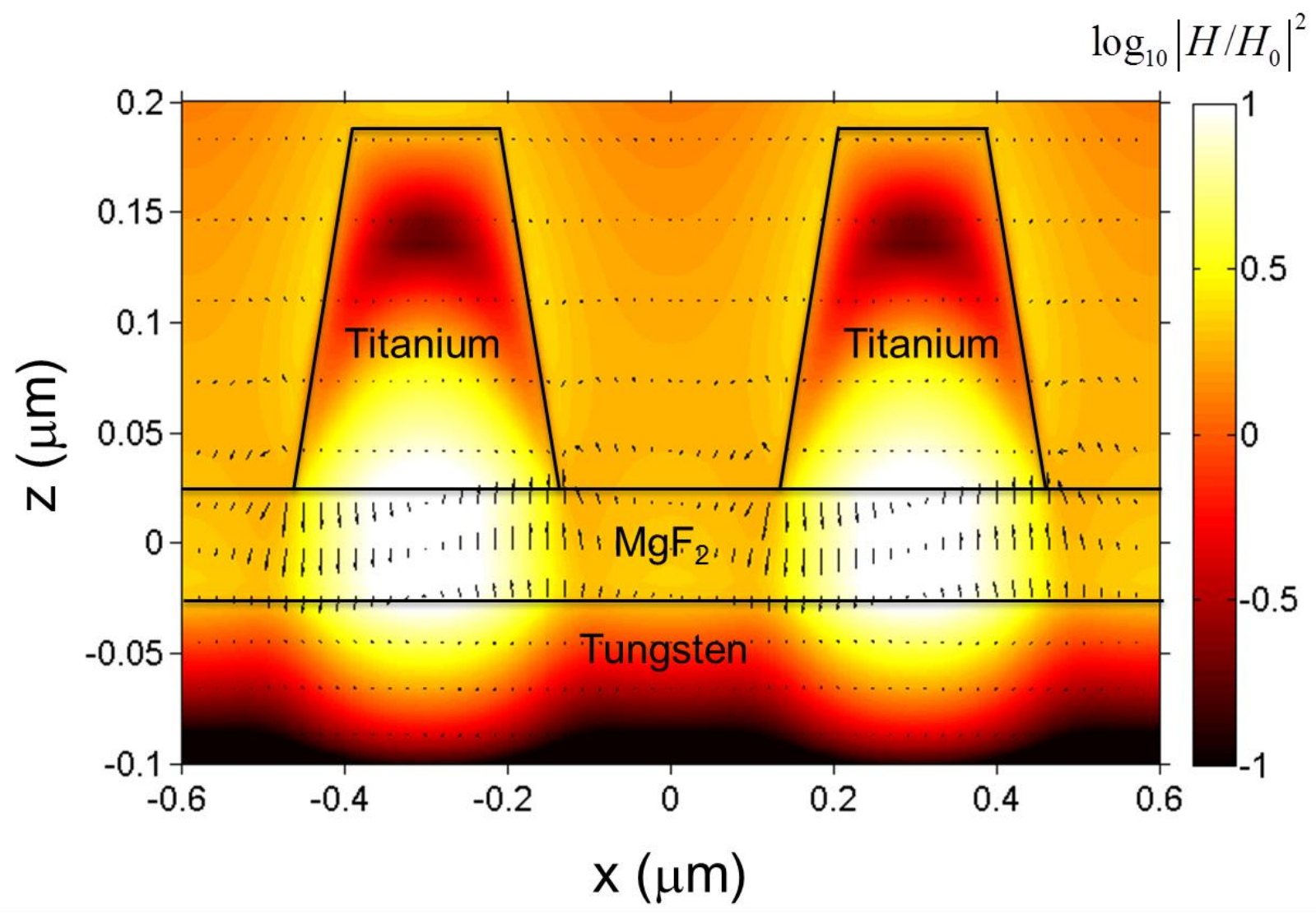

Wang et al., Figure 3 

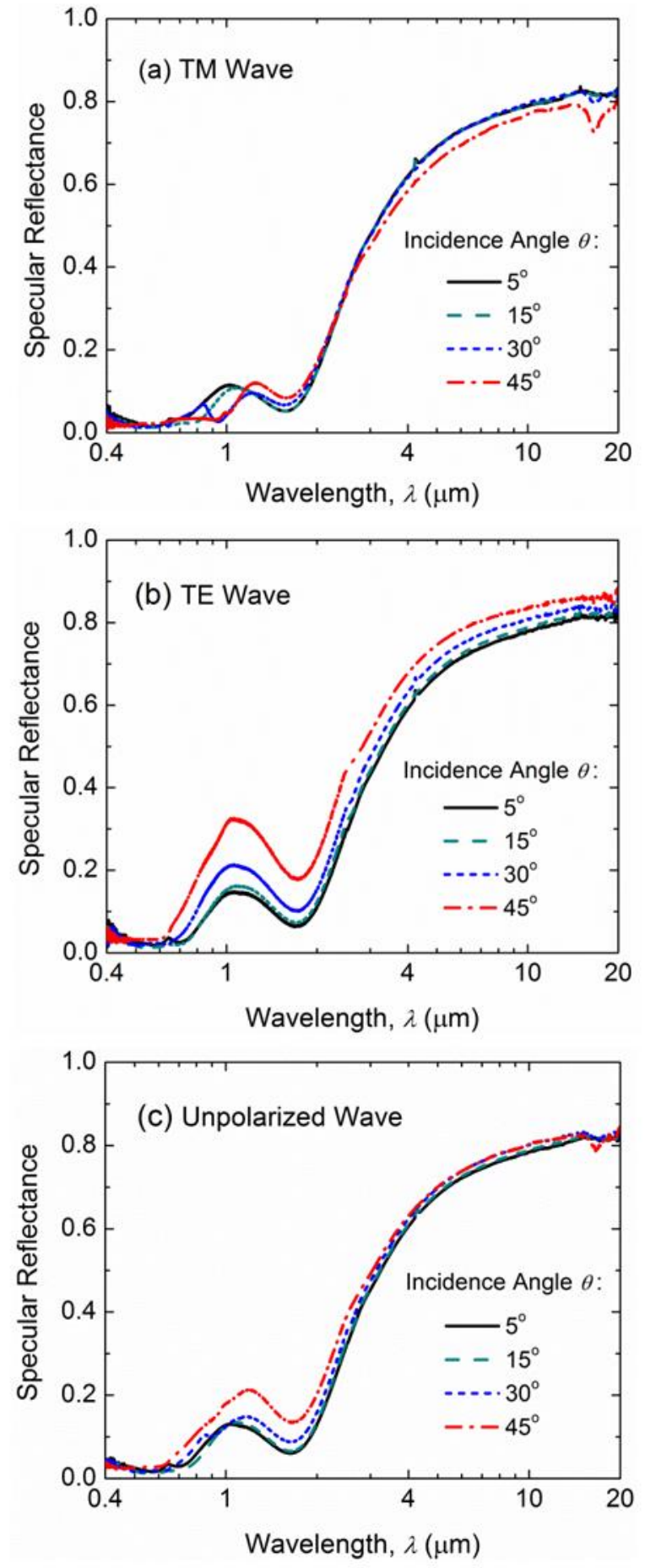

Wang et al., Figure 4 
(a)

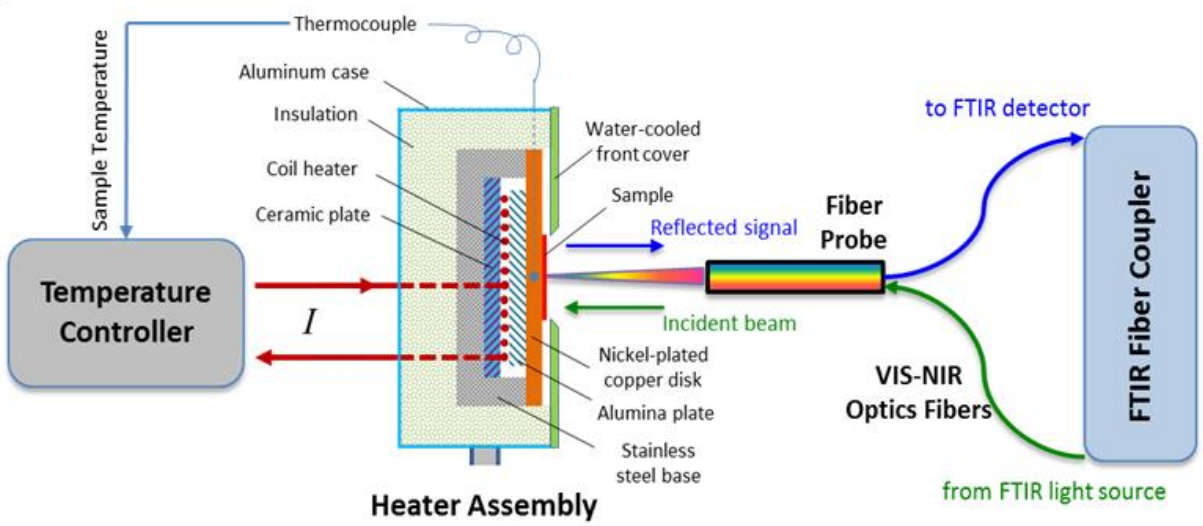

(b)

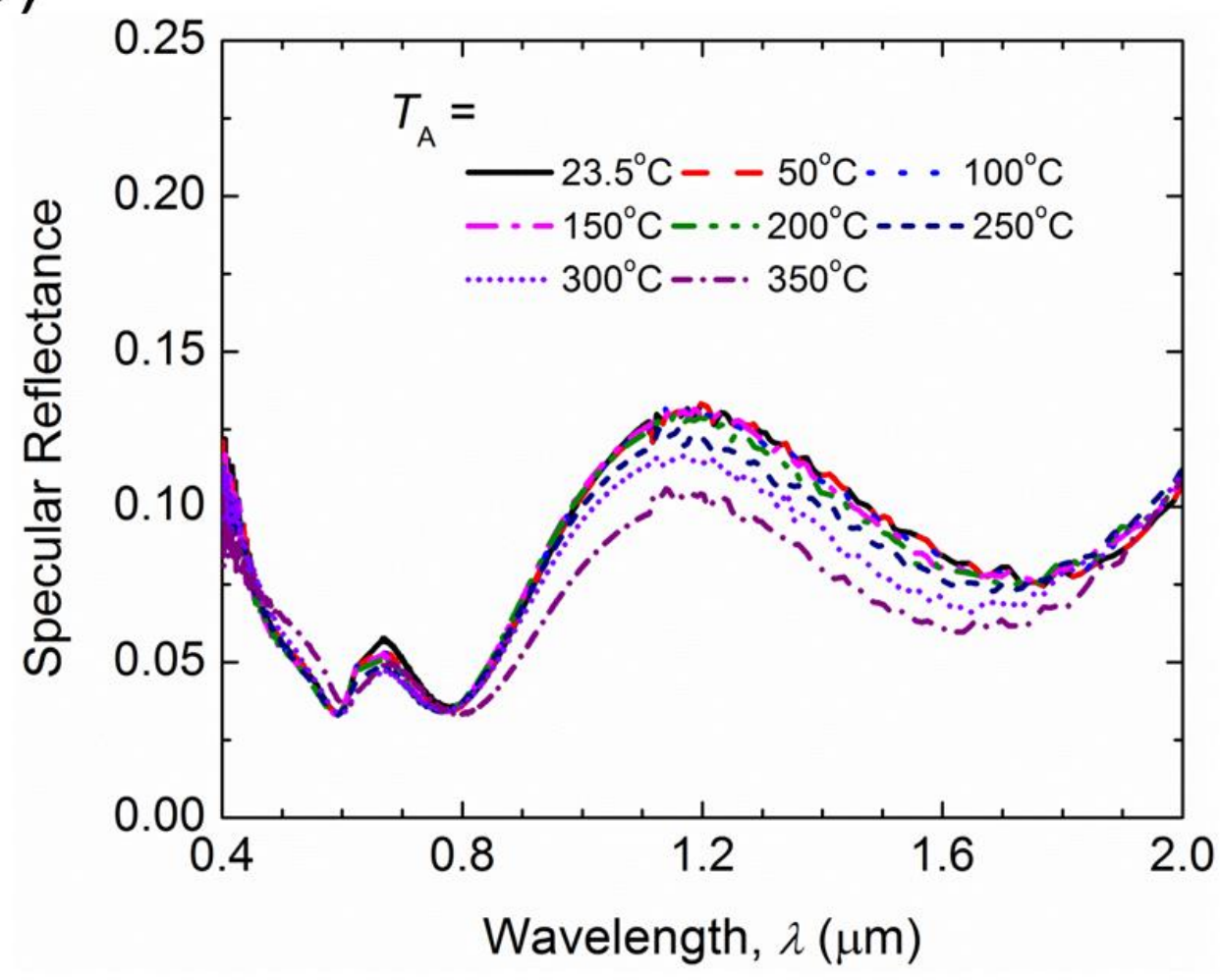

Wang et al., Figure 5 

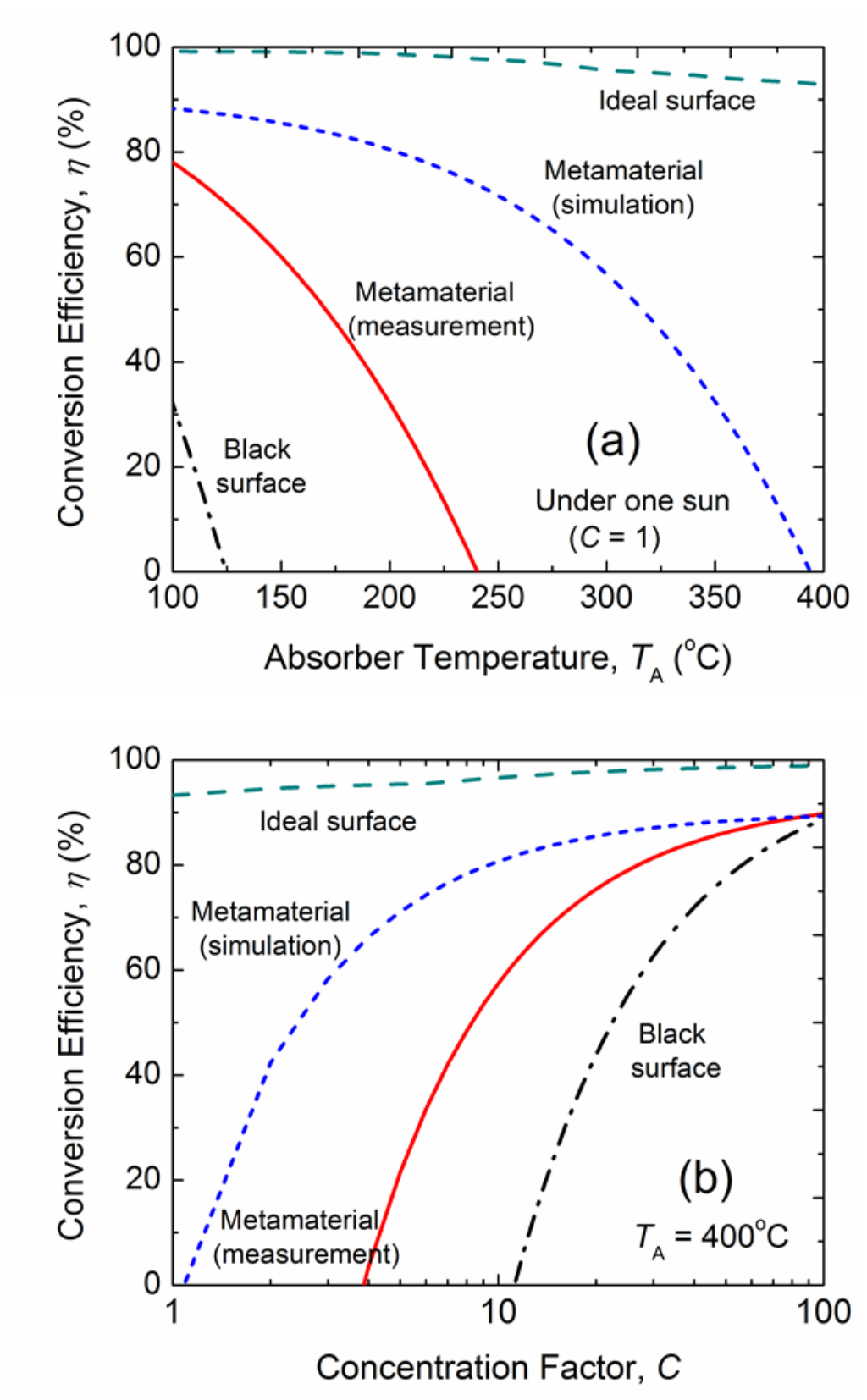

Wang et al., Figure 6 